\title{
Uniformly asymptotic stability of almost periodic solutions for a competitive system with impulsive perturbations
}

\author{
Ronghua Tan, Weifang Liu, Qinglong Wang and Zhijun Liu*
}

\section{"Correspondence:}

zhijun_liu47@hotmail.com

Department of Mathematics, Hubei

University for Nationalities, Enshi,

Hubei 445000, P.R. China

\begin{abstract}
Impulsive differential models play an important role in modeling population systems. In this article, we consider an almost periodic competitive model subject to impulsive perturbations and establish sufficient conditions for the uniformly asymptotic stability of a unique positive almost periodic solution for the system. The example and its numerical simulations are carried out to illustrate the feasibility of our main results.
\end{abstract}

Keywords: impulsive competitive system; positive almost periodic solutions; uniformly asymptotic stability; Lyapunov function

\section{Introduction}

In [1], Gopalsamy introduced the following autonomous two-species competitive system

$$
\left\{\begin{array}{l}
x_{1}^{\prime}(t)=x_{1}(t)\left[a_{1}-b_{1} x_{1}(t)-c_{1} x_{2}(t)-d_{1} x_{1}^{2}(t)\right] \\
x_{2}^{\prime}(t)=x_{2}(t)\left[a_{2}-b_{2} x_{2}(t)-c_{2} x_{1}(t)-d_{2} x_{2}^{2}(t)\right]
\end{array}\right.
$$

where $x_{1}(t), x_{2}(t)$ can be interpreted as the density of two competing species at time $t$, respectively. $a_{1}, a_{2}$ stand for the intrinsic growth rates of two species, $b_{1}, d_{1}, b_{2}, d_{2}$ represent the effects of intra-specific competition, and $c_{1}, c_{2}$ are the effects of inter-specific competition. Notice that the coefficients, in the real world, are not unchanged constants owing to the variation of environment, and the effect of a varying environment is significant for evolutionary theory as the selective forces on systems in such a fluctuating environment differ from those in a stable environment. So it is realistic to consider a corresponding non-autonomous version with the form

$$
\left\{\begin{array}{l}
x_{1}^{\prime}(t)=x_{1}(t)\left[a_{1}(t)-b_{1}(t) x_{1}(t)-c_{1}(t) x_{2}(t)-d_{1}(t) x_{1}^{2}(t)\right] \\
x_{2}^{\prime}(t)=x_{2}(t)\left[a_{2}(t)-b_{2}(t) x_{2}(t)-c_{2}(t) x_{1}(t)-d_{2}(t) x_{2}^{2}(t)\right] .
\end{array}\right.
$$

Here, all the coefficients $a_{i}(t), b_{i}(t), c_{i}(t), d_{i}(t)(i=1,2)$ are subject to fluctuation in time. Furthermore, it is known that the assumption of almost periodicity of the coefficients is a way of incorporating the time-dependent variability of the environment, and especially, if the various components of the environment are with incommensurable periods, then it is reasonable to consider the environment to be almost periodic, which leads to the almost periodicity of the coefficients of system (1.2). On the other hand, species live in a 
real fluctuating medium, and human exploitation activities might result in the duration of abrupt changes. Such changes can be well approximated as impulses, and these processes tend to be reasonably modeled by impulsive differential equations.

Motivated by the above facts, we establish the following almost periodic competitive system with impulsive perturbations:

$$
\left\{\begin{array}{l}
x_{1}^{\prime}(t)=x_{1}(t)\left[a_{1}(t)-b_{1}(t) x_{1}(t)-c_{1}(t) x_{2}(t)-d_{1}(t) x_{1}^{2}(t)\right], \\
x_{2}^{\prime}(t)=x_{2}(t)\left[a_{2}(t)-b_{2}(t) x_{2}(t)-c_{2}(t) x_{1}(t)-d_{2}(t) x_{2}^{2}(t)\right], \\
x_{1}\left(\tau_{k}^{+}\right)=\left(1+\gamma_{1 k}\right) x_{1}\left(\tau_{k}\right), \\
x_{2}\left(\tau_{k}^{+}\right)=\left(1+\gamma_{2 k}\right) x_{2}\left(\tau_{k}\right),
\end{array}\right\} \quad t=\tau_{k}, k \in \mathbb{N} .
$$

Here, $x_{1}\left(0^{+}\right)=x_{1}(0)>0, x_{2}\left(0^{+}\right)=x_{2}(0)>0, \mathbb{N}$ is the set of positive integers, the coefficients $a_{i}(t), b_{i}(t), c_{i}(t), d_{i}(t)$ are all continuous almost periodic functions which are bounded above and below by positive constants, $\gamma_{1 k}>-1$ and $\gamma_{2 k}>-1$ are constants and $0<\tau_{1}<\tau_{2}<\cdots<\tau_{k}<\tau_{k+1}<\cdots$ are impulse points with $\lim _{k \rightarrow+\infty} \tau_{k}=+\infty$. The jump conditions reflect the possibility of impulsive effects on two species. From biological viewpoints, when $\gamma_{i k}>0$, the perturbations may stand for stocking, while $\gamma_{i k}<0$ the perturbations mean harvesting.

In the research of population ecology, competitive systems are very important to describe the interactions in the multi-species population dynamics. Many competitive systems have been studied recently by many authors and there is quite extensive literature concerned with the dynamics such as stability of equilibrium [2], persistence [3], permanence or partial extinction [4-7], positive periodic solution [8-11], positive almost periodic solution [12-15] etc. However, there are not many papers considering the stability of positive almost periodic solutions for impulsive competitive systems [14-16]. In this article, we make an attempt to discuss such an issue by considering system (1.3). The rest of this paper is arranged as follows. In Section 2, we present some notations, definitions and lemmas. In Section 3, we give the main result on the uniformly asymptotic stability of a unique positive almost periodic solution for system (1.3). In Section 4, an example together with its numerical simulations is presented to verify the validity of the proposed criteria.

\section{Preliminaries}

In this section, we give some notations, definitions, lemmas which are useful for establishing our main result (i.e., Theorem 3.1).

Denote by $\mathbb{R}^{+}, \mathbb{R}$ and $\mathbb{Z}$ the sets of nonnegative real numbers, real numbers and integers, respectively. $\mathbb{R}^{2}$ and $\mathbb{R}^{n}$ denote the cone of a two-dimensional and $n$-dimensional real Euclidean space, respectively.

Definition 2.1 (see [17]) A sequence $\left\{\tau_{k}\right\}$ is called almost periodic if for any $\epsilon>0$ there exists a relatively dense set of its $\epsilon$-periods, i.e., there exists such a positive integer $N=$ $N(\epsilon)$ that, for any arbitrary $k \in \mathbb{Z}$, there is at least an integer $p$ in the segment $[k, k+N]$, for which $\left|\tau_{k+p}-\tau_{k}\right|<\epsilon$ holds.

Definition 2.2 (see [17]) The set of sequences $\left\{\tau_{k}^{j}=\tau_{k+j}-\tau_{k}\right\}, k, j \in \mathbb{Z}$, is said to be uniformly almost periodic if for arbitrary $\epsilon>0$, there exists a relatively dense set of $\epsilon$-almost periodic common for any sequences. 
Let $P C(\mathbb{R}, \mathbb{R})=\left\{u: \mathbb{R} \rightarrow \mathbb{R}: u\right.$ is continuous for $t \in \mathbb{R}, t \neq \tau_{k}$, continuous from the left for $t \in \mathbb{R}$ and discontinuities of the first kind occur at the point $\left.\tau_{k} \in \mathbb{R}, k \in \mathbb{N}\right\}$.

Definition 2.3 (see [17]) The function $\varphi \in P C(\mathbb{R}, \mathbb{R})$ is said to be almost periodic if the following conditions hold:

(1) The set of sequences $\left\{\tau_{k}^{j}\right\}, k, j \in \mathbb{Z}$ is uniformly almost periodic.

(2) For any $\epsilon>0$, there exists a positive number $\delta=\delta(\epsilon)$ such that if the points $t^{\prime}$ and $t^{\prime \prime}$ belong to the same interval of continuity and $\left|t^{\prime}-t^{\prime \prime}\right|<\delta$, then $\left|\varphi\left(t^{\prime}\right)-\varphi\left(t^{\prime \prime}\right)\right|<\epsilon$.

(3) For any $\epsilon>0$, there exists a relative dense set $T$ of $\epsilon$-almost periods such that if $\tau \in T$, then $|\varphi(t+\tau)-\varphi(t)|<\epsilon$ for all $t \in \mathbb{R}$, satisfying the condition $\left|t-\tau_{k}\right|>\epsilon$, $k \in \mathbb{Z}$.

Consider the following non-impulsive system which corresponds to system (1.3)

$$
\left\{\begin{array}{l}
y_{1}^{\prime}(t)=y_{1}(t)\left[a_{1}(t)-B_{1}(t) y_{1}(t)-C_{1}(t) y_{2}(t)-D_{1}(t) y_{1}^{2}(t)\right] \\
y_{2}^{\prime}(t)=y_{2}(t)\left[a_{2}(t)-B_{2}(t) y_{2}(t)-C_{2}(t) y_{1}(t)-D_{2}(t) y_{2}^{2}(t)\right]
\end{array}\right.
$$

where

$$
\begin{aligned}
& B_{i}(t)=b_{i}(t) \prod_{0<\tau_{k}<t}\left(1+\gamma_{i k}\right), \quad C_{i}(t)=c_{i}(t) \prod_{0<\tau_{k}<t}\left(1+\gamma_{j k}\right), \\
& D_{i}(t)=d_{i}(t) \prod_{0<\tau_{k}<t}\left(1+\gamma_{i k}\right)^{2}, \quad i, j=1,2, i \neq j .
\end{aligned}
$$

The following Lemma 2.1 is obvious.

Lemma 2.1 Any solution $\left(y_{1}(t), y_{2}(t)\right)$ of system (2.1) satisfies $y_{i}(t)>0$ for all $t \geq 0$.

Lemma 2.2 For systems (1.3) and (2.1), we have the following conclusions.

(1) If $\left(y_{1}(t), y_{2}(t)\right)$ is a solution of system (2.1), then $\left(x_{1}(t), x_{2}(t)\right)=\left(\prod_{0<\tau_{k}<t}\left(1+\gamma_{1 k}\right) y_{1}(t)\right.$, $\left.\prod_{0<\tau_{k}<t}\left(1+\gamma_{2 k}\right) y_{2}(t)\right)$ is a solution of system (1.3).

(2) If $\left(x_{1}(t), x_{2}(t)\right)$ is a solution of system (1.3), then $\left(y_{1}(t), y_{2}(t)\right)=\left(\prod_{0<\tau_{k}<t}\left(1+\gamma_{1 k}\right)^{-1} x_{1}(t)\right.$, $\left.\prod_{0<\tau_{k}<t}\left(1+\gamma_{2 k}\right)^{-1} x_{2}(t)\right)$ is a solution of system (2.1).

Proof (1) Assume that $\left(y_{1}(t), y_{2}(t)\right)$ is a solution of system (2.1). It is easy to see that $x_{i}(t)=$ $\prod_{0<\tau_{k}<t}\left(1+\gamma_{i k}\right) y_{i}(t)$ are continuous on the interval $\left(\tau_{k}, \tau_{k+1}\right]$, then for any $t \neq \tau_{k}, k \in \mathbb{N}$, one has

$$
\begin{aligned}
x_{1}^{\prime}(t) & -x_{1}(t)\left[a_{1}(t)-b_{1}(t) x_{1}(t)-c_{1}(t) x_{2}(t)-d_{1}(t) x_{1}^{2}(t)\right] \\
= & \prod_{0<\tau_{k}<t}\left(1+\gamma_{1 k}\right)\left\{y_{1}^{\prime}(t)-y_{1}(t)\left[a_{1}(t)-b_{1}(t) \prod_{0<\tau_{k}<t}\left(1+\gamma_{1 k}\right) y_{1}(t)\right.\right. \\
& \left.\left.\quad-c_{1}(t) \prod_{0<\tau_{k}<t}\left(1+\gamma_{2 k}\right) y_{2}(t)-d_{1}(t) \prod_{0<\tau_{k}<t}\left(1+\gamma_{1 k}\right)^{2} y_{1}^{2}(t)\right]\right\} \\
= & \prod_{0<\tau_{k}<t}\left(1+\gamma_{1 k}\right)\left\{y_{1}^{\prime}(t)-y_{1}(t)\left[a_{1}(t)-B_{1}(t) y_{1}(t)-C_{1}(t) y_{2}(t)-D_{1}(t) y_{1}^{2}(t)\right]\right\} \\
= & 0
\end{aligned}
$$


and

$$
\begin{aligned}
x_{2}^{\prime}(t) & -x_{2}(t)\left[a_{2}(t)-b_{2}(t) x_{2}(t)-c_{2}(t) x_{1}(t)-d_{2}(t) x_{2}^{2}(t)\right] \\
= & \prod_{0<\tau_{k}<t}\left(1+\gamma_{2 k}\right)\left\{y_{2}^{\prime}(t)-y_{2}(t)\left[a_{2}(t)-b_{2}(t) \prod_{0<\tau_{k}<t}\left(1+\gamma_{2 k}\right) y_{2}(t)\right.\right. \\
& \left.\left.\quad-c_{2}(t) \prod_{0<\tau_{k}<t}\left(1+\gamma_{1 k}\right) y_{1}(t)-d_{2}(t) \prod_{0<\tau_{k}<t}\left(1+\gamma_{2 k}\right)^{2} y_{2}^{2}(t)\right]\right\} \\
= & \prod_{0<\tau_{k}<t}\left(1+\gamma_{2 k}\right)\left\{y_{2}^{\prime}(t)-y_{2}(t)\left[a_{2}(t)-B_{2}(t) y_{2}(t)-C_{2}(t) y_{1}(t)-D_{2}(t) y_{2}^{2}(t)\right]\right\} \\
= & 0 .
\end{aligned}
$$

On the other hand, for every $t=\tau_{k}, k \in \mathbb{N}$, we get

$$
\begin{aligned}
x_{1}\left(\tau_{k}^{+}\right) & =\lim _{t \rightarrow \tau_{k}^{+}} \prod_{0<\tau_{k}<t}\left(1+\gamma_{1 k}\right) y_{1}(t)=\prod_{0<\tau_{j} \leq \tau_{k}}\left(1+\gamma_{1 j}\right) y_{1}\left(\tau_{k}\right) \\
& =\left(1+\gamma_{1 k}\right) \prod_{0<\tau_{j}<\tau_{k}}\left(1+\gamma_{1 j}\right) y_{1}\left(\tau_{k}\right)=\left(1+\gamma_{1 k}\right) x_{1}\left(\tau_{k}\right)
\end{aligned}
$$

and

$$
\begin{aligned}
x_{2}\left(\tau_{k}^{+}\right) & =\lim _{t \rightarrow \tau_{k}^{+}} \prod_{0<\tau_{k}<t}\left(1+\gamma_{2 k}\right) y_{2}(t)=\prod_{0<\tau_{j} \leq \tau_{k}}\left(1+\gamma_{2 j}\right) y_{2}\left(\tau_{k}\right) \\
& =\left(1+\gamma_{2 k}\right) \prod_{0<\tau_{j}<\tau_{k}}\left(1+\gamma_{2 j}\right) y_{2}\left(\tau_{k}\right)=\left(1+\gamma_{2 k}\right) x_{2}\left(\tau_{k}\right) .
\end{aligned}
$$

Thus $\left(x_{1}(t), x_{2}(t)\right)$ is a solution of system (1.3).

(2) Since $y_{1}(t)$ and $y_{2}(t)$ are continuous on each interval $\left(\tau_{k}, \tau_{k+1}\right]$. From system (2.1), one can easily check the continuity of $y_{i}(t)$ at the impulse points $t=\tau_{k}, k \in \mathbb{N}$. Recalling system (1.3), we have

$$
\begin{aligned}
& y_{1}\left(\tau_{k}^{+}\right)=\prod_{0<\tau_{j} \leq \tau_{k}}\left(1+\gamma_{1 j}\right)^{-1} x_{1}\left(\tau_{k}^{+}\right)=\prod_{0<\tau_{j}<\tau_{k}}\left(1+\gamma_{1 j}\right)^{-1} x_{1}\left(\tau_{k}\right)=y_{1}\left(\tau_{k}\right), \\
& y_{2}\left(\tau_{k}^{+}\right)=\prod_{0<\tau_{j} \leq \tau_{k}}\left(1+\gamma_{2 j}\right)^{-1} x_{2}\left(\tau_{k}^{+}\right)=\prod_{0<\tau_{j}<\tau_{k}}\left(1+\gamma_{2 j}\right)^{-1} x_{2}\left(\tau_{k}\right)=y_{2}\left(\tau_{k}\right) .
\end{aligned}
$$

Also, by the basic theory of impulsive differential equations in $[18,19]$, we know that $x_{i}\left(\tau_{k}^{-}\right)=x_{i}\left(\tau_{k}\right)$. So we get

$$
\begin{aligned}
& y_{1}\left(\tau_{k}^{-}\right)=\prod_{0<\tau_{j}<\tau_{k}}\left(1+\gamma_{1 j}\right)^{-1} x_{1}\left(\tau_{k}^{-}\right)=\prod_{0<\tau_{j}<\tau_{k}}\left(1+\gamma_{1 j}\right)^{-1} x_{1}\left(\tau_{k}\right)=y_{1}\left(\tau_{k}\right), \\
& y_{2}\left(\tau_{k}^{-}\right)=\prod_{0<\tau_{j}<\tau_{k}}\left(1+\gamma_{2 j}\right)^{-1} x_{2}\left(\tau_{k}^{-}\right)=\prod_{0<\tau_{j}<\tau_{k}}\left(1+\gamma_{2 j}\right)^{-1} x_{2}\left(\tau_{k}\right)=y_{2}\left(\tau_{k}\right) .
\end{aligned}
$$

Equations (2.7) and (2.8) imply that $y_{1}(t)$ and $y_{2}(t)$ are continuous on $\mathbb{R}^{+}$. It is easy to see that $\left(y_{1}(t), y_{2}(t)\right)$ is a solution of system (2.1). The proof of Lemma 2.2 is complete. 
Consider the following differential equation:

$$
X^{\prime}=f(t, X), \quad t \in \mathbb{R}^{+},
$$

where $f(t, X) \in C\left(\mathbb{R}^{+} \times \mathbb{D}, \mathbb{R}^{n}\right)$ and $\mathbb{D}$ is an open set in $\mathbb{R}^{n}, f(t, X)$ is almost periodic in $t$ uniformly with respect to $X \in \mathbb{D}$. The following associate product system of system (2.9) can be expressed as

$$
X^{\prime}=f(t, X), \quad Y^{\prime}=f(t, Y) .
$$

Lemma 2.3 (see Theorem 6.3 in [20]) Suppose that there exists a Lyapunov function $V(t, X, Y)$ defined on $[0,+\infty) \times \mathbb{D} \times \mathbb{D}$, which satisfies the following conditions:

(1) $a(\|X-Y\|) \leq V(t, X, Y) \leq b(\|X-Y\|)$, where $a(\kappa)$ and $b(\kappa)$ are continuous, increasing and positive definite functions.

(2) $\left|V\left(t, X_{1}, Y_{1}\right)-V\left(t, X_{2}, Y_{2}\right)\right| \leq K\left\{\left\|X_{1}-X_{2}\right\|+\left\|Y_{1}-Y_{2}\right\|\right\}$, where $K>0$ is a constant.

(3) $V_{(2.10)}^{\prime}(t, X, Y) \leq-\mu V(t, X, Y)$, where $\mu>0$ is a constant.

Moreover, suppose that system (2.9) has a solution that remains in a compact set $\mathbb{S} \subset \mathbb{D}$ for all $t \geq 0$. Then system (2.9) has a unique almost periodic solution in $\mathbb{S}$, which is uniformly asymptotically stable in $\mathbb{D}$.

Lemma 2.4 (see [21]) (1) If $a>0, b>0$ and $x^{\prime}(t) \geq x(t)(a-b x(t))$, when $t \geq 0$ and $x(0)>0$, we have $\liminf _{t \rightarrow+\infty} x(t) \geq a / b$. (2) If $a>0, b>0$ and $x^{\prime}(t) \leq x(t)(a-b x(t))$, when $t \geq 0$ and $x(0)>0$, we have $\lim \sup _{t \rightarrow+\infty} x(t) \leq a / b$.

For convenience, given an almost periodic function $g(t)$ defined on $\mathbb{R}^{+}$, let $g^{L}$ and $g^{U}$ be defined as $g^{L}=\inf _{t \in \mathbb{R}^{+}} g(t), g^{U}=\sup _{t \in \mathbb{R}^{+}} g(t)$.

\section{Lemma 2.5 Assume that the following two conditions}

(A1) there exist positive constants $\alpha_{i}, \beta_{i}$ such that $\alpha_{i} \leq \prod_{0<\tau_{k}<t}\left(1+\gamma_{i k}\right) \leq \beta_{i}, i=1,2$,

(A2) $a_{1}^{L}-c_{1}^{U} \beta_{2} M_{2}>0$ and $a_{2}^{L}-c_{2}^{U} \beta_{1} M_{1}>0$

hold, then any solution $\left(y_{1}(t), y_{2}(t)\right)$ of system (2.1) satisfies

$$
m_{i} \leq \liminf _{t \rightarrow+\infty} y_{i}(t) \leq \limsup _{t \rightarrow+\infty} y_{i}(t) \leq M_{i}
$$

where $m_{i}=\left(a_{i}^{L}-c_{i}^{U} \beta_{j} M_{j}\right) /\left(b_{i}^{U} \beta_{i}+d_{i}^{U} \beta_{i}^{2} M_{i}\right), M_{i}=a_{i}^{U} /\left(b_{i}^{L} \alpha_{i}\right), 1 \leq i, j \leq 2 ; i \neq j$.

Proof Let $\left(y_{1}(t), y_{2}(t)\right)$ be any solution of system (2.1). It follows from system (2.1) and (A1) that we have

$$
y_{i}^{\prime}(t) \leq y_{i}(t)\left[a_{i}(t)-B_{i}(t) y_{i}(t)\right] \leq y_{i}(t)\left[a_{i}^{U}-b_{i}^{L} \alpha_{i} y_{i}(t)\right] .
$$

Using (2) in Lemma 2.4, one has

$$
\limsup _{t \rightarrow+\infty} y_{i}(t) \leq a_{i}^{U} /\left(b_{i}^{L} \alpha_{i}\right) \stackrel{\text { def }}{=} M_{i} .
$$

Hence, for any small constant $\varepsilon>0$, there exists $T_{0}>0$ such that for $t \geq T_{0}$,

$$
y_{i}(t) \leq M_{i}+\varepsilon, \quad i=1,2 .
$$


Together with system (2.1), we can derive that

$$
y_{i}^{\prime}(t) \geq y_{i}(t)\left[a_{i}^{L}-c_{i}^{U} \beta_{j}\left(M_{j}+\varepsilon\right)-\left(b_{i}^{U} \beta_{i}+d_{i}^{U} \beta_{i}^{2}\left(M_{i}+\varepsilon\right)\right) y_{i}(t)\right], \quad i, j=1,2, i \neq j .
$$

Thus from (A2), (1) in Lemma 2.4 and $\varepsilon>0$ is arbitrarily small, one has

$$
\liminf _{t \rightarrow+\infty} y_{i}(t) \geq\left(a_{i}^{L}-c_{i}^{U} \beta_{j} M_{j}\right) /\left(b_{i}^{U} \beta_{i}+d_{i}^{U} \beta_{i}^{2} M_{i}\right) \stackrel{\text { def }}{=} m_{i}, \quad i, j=1,2, i \neq j
$$

The proof of Lemma 2.5 is complete.

By (2.12) and (2.13), we denote by $\Theta$ the set of all solutions $\left(y_{1}(t), y_{2}(t)\right)$ of system $(2.1)$ satisfying $m_{i} \leq y_{i}(t) \leq M_{i}$, that is,

$$
\Theta=\left\{\left(y_{1}(t), y_{2}(t)\right) \mid m_{i} \leq y_{i}(t) \leq M_{i}, i=1,2\right\}
$$

Lemma 2.6 Assume that (A1) and (A2) are satisfied. Suppose further that

(A3) the set of sequences $\left\{\tau_{k}^{j}=\tau_{k+j}-\tau_{k}\right\}, k, j \in \mathbb{Z}$ is uniformly almost periodic,

(A4) $\prod_{0<\tau_{k}<t}\left(1+\gamma_{i k}\right)$ is an almost periodic function.

Then $\Theta \neq \phi$.

Proof The almost periodicity of $\left\{a_{i}(t)\right\},\left\{B_{i}(t)\right\},\left\{C_{i}(t)\right\},\left\{D_{i}(t)\right\}$ implies that there exists a sequence $\left\{t_{n}\right\}, t_{n} \rightarrow+\infty$ as $n \rightarrow+\infty$ such that

$$
\begin{array}{ll}
a_{i}\left(t+t_{n}\right) \rightarrow a_{i}(t), \quad & B_{i}\left(t+t_{n}\right) \rightarrow B_{i}(t), \\
C_{i}\left(t+t_{n}\right) \rightarrow C_{i}(t), & D_{i}\left(t+t_{n}\right) \rightarrow D_{i}(t), \quad i=1,2,
\end{array}
$$

as $n \rightarrow+\infty$ for $t \in \mathbb{R}^{+}$. It follows from (2.11) that, for any small enough $\varepsilon>0$, there exists $T_{1}>0$ such that for $t \geq T_{1}$,

$$
m_{i}-\varepsilon \leq y_{i}(t) \leq M_{i}+\varepsilon \text {. }
$$

It is obvious that the sequence $\left\{y_{i}\left(t+t_{n}\right)\right\}$ is uniformly bounded and equicontinuous on each bounded subset of $\mathbb{R}^{+}, i=1,2$. By Ascoli's theorem, we obtain that $\left\{y_{i}\left(t+t_{n}\right)\right\}$ exists a subsequence, also denoted by $\left\{y_{i}\left(t+t_{n}\right)\right\}$, converging on each bounded subset of $\mathbb{R}^{+}$as $n \rightarrow+\infty$. Therefore, there is a continuous function $\bar{y}_{i}(t)$ satisfying

$$
y_{i}\left(t+t_{n}\right) \rightarrow \bar{y}_{i}(t) \quad \text { for } t \in \mathbb{R}^{+} \text {as } n \rightarrow+\infty .
$$

For any $T_{2} \in \mathbb{R}^{+}$such that $t_{n}+T_{2} \geq T_{1}$ for large enough $n$. Let $t \geq 0$, we have

$$
\left\{\begin{aligned}
y_{1}\left(t+t_{n}+T_{2}\right)= & y_{1}\left(t_{n}+T_{2}\right)+\int_{T_{2}}^{t+T_{2}} y_{1}\left(s+t_{n}\right)\left[a_{1}\left(s+t_{n}\right)-B_{1}\left(s+t_{n}\right) y_{1}\left(s+t_{n}\right)\right. \\
& \left.-C_{1}\left(s+t_{n}\right) y_{2}\left(s+t_{n}\right)-D_{1}\left(s+t_{n}\right) y_{1}^{2}\left(s+t_{n}\right)\right] d s, \\
y_{2}\left(t+t_{n}+T_{2}\right)= & y_{2}\left(t_{n}+T_{2}\right)+\int_{T_{2}}^{t+T_{2}} y_{2}\left(s+t_{n}\right)\left[a_{2}\left(s+t_{n}\right)-B_{2}\left(s+t_{n}\right) y_{2}\left(s+t_{n}\right)\right. \\
& \left.-C_{2}\left(s+t_{n}\right) y_{1}\left(s+t_{n}\right)-D_{2}\left(s+t_{n}\right) y_{2}^{2}\left(s+t_{n}\right)\right] d s .
\end{aligned}\right.
$$


Using Lebesgue' dominated convergence theorem, and letting $n \rightarrow+\infty$ in (2.17), one has

$$
\left\{\begin{aligned}
\bar{y}_{1}\left(t+T_{2}\right)= & \bar{y}_{1}\left(T_{2}\right)+\int_{T_{2}}^{t+T_{2}} \bar{y}_{1}(s)\left[a_{1}(s)-B_{1}(s) \bar{y}_{1}(s)\right. \\
& \left.-C_{1}(s) \bar{y}_{2}(s)-D_{1}(s) \bar{y}_{1}^{2}(s)\right] d s \\
\bar{y}_{2}\left(t+T_{2}\right)= & \bar{y}_{2}\left(T_{2}\right)+\int_{T_{2}}^{t+T_{2}} \bar{y}_{2}(s)\left[a_{2}(s)-B_{2}(s) \bar{y}_{2}(s)\right. \\
& \left.-C_{2}(s) \bar{y}_{1}(s)-D_{2}(s) \bar{y}_{2}^{2}(s)\right] d s
\end{aligned}\right.
$$

Since $T_{2} \in \mathbb{R}^{+}$is arbitrary, $\left(\bar{y}_{1}(t), \bar{y}_{2}(t)\right)$ is a solution of system (2.1) on $\mathbb{R}^{+}$. We easily obtain that $m_{i}-\varepsilon \leq \bar{y}_{i}(t) \leq M_{i}+\varepsilon$ for $t \in \mathbb{R}^{+}, i=1,2$. Furthermore, since $\varepsilon$ is arbitrarily small, we get that $m_{i} \leq \bar{y}_{i}(t) \leq M_{i}, i=1,2$, for $t \in \mathbb{R}^{+}$. The proof of Lemma 2.6 is complete.

\section{The main result}

In this section, we give our main result and establish the uniformly asymptotic stability of a unique positive almost periodic solution for system (1.3).

Theorem 3.1 Assume that (A1)-(A4) hold. Furthermore, assume that

(A5) there exist positive constants $\theta_{1}, \theta_{2}, \sigma$, where $\sigma=\min \left\{s_{1}, s_{2}\right\}$, and

$$
\begin{aligned}
& s_{1}=\theta_{1} b_{1}^{L} \alpha_{1} m_{1}+2 \theta_{1} d_{1}^{L} \alpha_{1}^{2} m_{1}^{2}-\theta_{2} c_{2}^{U} \beta_{1} M_{1}, \\
& s_{2}=\theta_{2} b_{2}^{L} \alpha_{2} m_{2}+2 \theta_{2} d_{2}^{L} \alpha_{2}^{2} m_{2}^{2}-\theta_{1} c_{1}^{U} \beta_{2} M_{2} .
\end{aligned}
$$

Then system (1.3) has a unique uniformly asymptotically stable positive almost periodic solution.

Proof Let us make the change of variables

$$
z_{1}(t)=\ln y_{1}(t), \quad z_{2}(t)=\ln y_{2}(t)
$$

then system (2.1) can be rewritten as

$$
\left\{\begin{array}{l}
z_{1}^{\prime}(t)=a_{1}(t)-B_{1}(t) \exp \left\{z_{1}(t)\right\}-C_{1}(t) \exp \left\{z_{2}(t)\right\}-D_{1}(t) \exp \left\{2 z_{1}(t)\right\} \\
z_{2}^{\prime}(t)=a_{2}(t)-B_{2}(t) \exp \left\{z_{2}(t)\right\}-C_{2}(t) \exp \left\{z_{1}(t)\right\}-D_{2}(t) \exp \left\{2 z_{2}(t)\right\}
\end{array}\right.
$$

Obviously, the existence of a unique almost periodic solution of system (2.1) is equivalent to that of system (3.2). By Lemma 2.6, there is a bounded solution $\left(z_{1}(t), z_{2}(t)\right)$ of system (3.2) satisfying

$$
\ln m_{1} \leq z_{1}(t) \leq \ln M_{1}, \quad \ln m_{2} \leq z_{2}(t) \leq \ln M_{2}
$$

Define the norm $\left\|\left(z_{1}(t), z_{2}(t)\right)\right\|=\left|z_{1}(t)\right|+\left|z_{2}(t)\right|$, where $\left(z_{1}(t), z_{2}(t)\right) \in \mathbb{R}^{2}$. Consider the associate product system of system (3.2)

$$
\left\{\begin{array}{l}
z_{1}^{\prime}(t)=a_{1}(t)-B_{1}(t) \exp \left\{z_{1}(t)\right\}-C_{1}(t) \exp \left\{z_{2}(t)\right\}-D_{1}(t) \exp \left\{2 z_{1}(t)\right\}, \\
z_{2}^{\prime}(t)=a_{2}(t)-B_{2}(t) \exp \left\{z_{2}(t)\right\}-C_{2}(t) \exp \left\{z_{1}(t)\right\}-D_{2}(t) \exp \left\{2 z_{2}(t)\right\}, \\
w_{1}^{\prime}(t)=a_{1}(t)-B_{1}(t) \exp \left\{w_{1}(t)\right\}-C_{1}(t) \exp \left\{w_{2}(t)\right\}-D_{1}(t) \exp \left\{2 w_{1}(t)\right\} \\
w_{2}^{\prime}(t)=a_{2}(t)-B_{2}(t) \exp \left\{w_{2}(t)\right\}-C_{2}(t) \exp \left\{w_{1}(t)\right\}-D_{2}(t) \exp \left\{2 w_{2}(t)\right\}
\end{array}\right.
$$


Here, $Z(t)=\left(z_{1}(t), z_{2}(t)\right)$ and $W(t)=\left(w_{1}(t), w_{2}(t)\right)$ are any two solutions of system (3.2) defined on $\mathbb{S}$, and $\mathbb{S}=\left\{\left(z_{1}(t), z_{2}(t)\right) \in \mathbb{R}^{2} \mid \ln m_{i} \leq z_{i}(t) \leq \ln M_{i}, i=1,2, t \in \mathbb{R}^{+}\right\}$.

Next, let us consider a Lyapunov function defined on $\mathbb{R}^{+} \times \mathbb{S} \times \mathbb{S}$ as follows:

$$
V(t, Z(t), W(t))=\theta_{1}\left|z_{1}(t)-w_{1}(t)\right|+\theta_{2}\left|z_{2}(t)-w_{2}(t)\right| .
$$

It is obvious that

$$
\min \left\{\theta_{1}, \theta_{2}\right\}\|Z(t)-W(t)\| \leq V(t, Z(t), W(t)) \leq \max \left\{\theta_{1}, \theta_{2}\right\}\|Z(t)-W(t)\| .
$$

Let $a(\kappa)=\min \left\{\theta_{1}, \theta_{2}\right\} \kappa, b(\kappa)=\max \left\{\theta_{1}, \theta_{2}\right\} \kappa$, then condition (1) in Lemma 2.3 is satisfied.

In addition, for any $(t, Z, W),(t, \tilde{Z}, \tilde{W}) \in \mathbb{R}^{+} \times \mathbb{S} \times \mathbb{S}$, one has

$$
\begin{aligned}
& |V(t, Z(t), W(t))-V(t, \tilde{Z}(t), \tilde{W}(t))| \\
& \quad=\left|\theta_{1}\right| z_{1}(t)-w_{1}(t)\left|+\theta_{2}\right| z_{2}(t)-w_{2}(t)\left|-\theta_{1}\right| \tilde{z}_{1}(t)-\tilde{w}_{1}(t)\left|-\theta_{2}\right| \tilde{z}_{2}(t)-\tilde{w}_{2}(t)|| \\
& \quad \leq \theta_{1}\left|z_{1}(t)-\tilde{z}_{1}(t)\right|+\theta_{1}\left|w_{1}(t)-\tilde{w}_{1}(t)\right|+\theta_{2}\left|z_{2}(t)-\tilde{z}_{2}(t)\right|+\theta_{2}\left|w_{2}(t)-\tilde{w}_{2}(t)\right| \\
& \quad \leq \max \left\{\theta_{1}, \theta_{2}\right\}\{\|Z(t)-\tilde{Z}(t)\|+\|W(t)-\tilde{W}(t)\|\} \\
& \quad=\lambda\{\|Z(t)-\tilde{Z}(t)\|+\|W(t)-\tilde{W}(t)\|\},
\end{aligned}
$$

where $\tilde{Z}(t)=\left(\tilde{z}_{1}(t), \tilde{z}_{2}(t)\right), \tilde{W}(t)=\left(\tilde{w}_{1}(t), \tilde{w}_{2}(t)\right), \lambda=\max \left\{\theta_{1}, \theta_{2}\right\}$. Hence, condition (2) in Lemma 2.3 is satisfied.

Finally, calculating the right derivative $D^{+} V(t)$ of $V(t)$ along the solutions of system (3.3), one has

$$
\begin{aligned}
D_{(3.3)}^{+} V(t)= & \theta_{1} \operatorname{sgn}\left(z_{1}(t)-w_{1}(t)\right)\left(z_{1}^{\prime}(t)-w_{1}^{\prime}(t)\right) \\
& +\theta_{2} \operatorname{sgn}\left(z_{2}(t)-w_{2}(t)\right)\left(z_{2}^{\prime}(t)-w_{2}^{\prime}(t)\right) \\
= & \theta_{1} \operatorname{sgn}\left(z_{1}(t)-w_{1}(t)\right)\left[-B_{1}(t)\left(\exp \left\{z_{1}(t)\right\}-\exp \left\{w_{1}(t)\right\}\right)\right. \\
& \left.-C_{1}(t)\left(\exp \left\{z_{2}(t)\right\}-\exp \left\{w_{2}(t)\right\}\right)-D_{1}(t)\left(\exp \left\{2 z_{1}(t)\right\}-\exp \left\{2 w_{1}(t)\right\}\right)\right] \\
& +\theta_{2} \operatorname{sgn}\left(z_{2}(t)-w_{2}(t)\right)\left[-B_{2}(t)\left(\exp \left\{z_{2}(t)\right\}-\exp \left\{w_{2}(t)\right\}\right)\right. \\
& -C_{2}(t)\left(\exp \left\{z_{1}(t)\right\}-\exp \left\{w_{1}(t)\right\}\right) \\
& \left.-D_{2}(t)\left(\exp \left\{2 z_{2}(t)\right\}-\exp \left\{2 w_{2}(t)\right\}\right)\right] .
\end{aligned}
$$

By the mean-value theorem, we have

$$
\begin{aligned}
& \exp \left\{z_{i}(t)\right\}-\exp \left\{w_{i}(t)\right\}=\xi_{i}(t)\left(z_{i}(t)-w_{i}(t)\right), \\
& \exp \left\{2 z_{i}(t)\right\}-\exp \left\{2 w_{i}(t)\right\}=2 \eta_{i}^{2}(t)\left(z_{i}(t)-w_{i}(t)\right),
\end{aligned}
$$

$i=1,2$, where $\xi_{i}(t)$ and $\eta_{i}(t)$ lie between $\exp \left\{z_{i}(t)\right\}$ and $\exp \left\{w_{i}(t)\right\}$, respectively. Substituting (3.8) into (3.7), one has

$$
\begin{aligned}
D_{(3.3)}^{+} V(t)= & \theta_{1} \operatorname{sgn}\left(z_{1}(t)-w_{1}(t)\right)\left[-B_{1}(t) \xi_{1}(t)\left(z_{1}(t)-w_{1}(t)\right)\right. \\
& \left.-C_{1}(t) \xi_{2}(t)\left(z_{2}(t)-w_{2}(t)\right)-2 D_{1}(t) \eta_{1}^{2}(t)\left(z_{1}(t)-w_{1}(t)\right)\right]
\end{aligned}
$$




$$
\begin{aligned}
& +\theta_{2} \operatorname{sgn}\left(z_{2}(t)-w_{2}(t)\right)\left[-B_{2}(t) \xi_{2}(t)\left(z_{2}(t)-w_{2}(t)\right)\right. \\
& \left.-C_{2}(t) \xi_{1}(t)\left(z_{1}(t)-w_{1}(t)\right)-2 D_{2}(t) \eta_{2}^{2}(t)\left(z_{2}(t)-w_{2}(t)\right)\right] \\
\leq & -\theta_{1} B_{1}(t) \xi_{1}(t)\left|z_{1}(t)-w_{1}(t)\right|+\theta_{1} C_{1}(t) \xi_{2}(t)\left|z_{2}(t)-w_{2}(t)\right| \\
& -2 \theta_{1} D_{1}(t) \eta_{1}^{2}(t)\left|z_{1}(t)-w_{1}(t)\right| \\
& -\theta_{2} B_{2}(t) \xi_{2}(t)\left|z_{2}(t)-w_{2}(t)\right|+\theta_{2} C_{2}(t) \xi_{1}(t)\left|z_{1}(t)-w_{1}(t)\right| \\
& -2 \theta_{2} D_{2}(t) \eta_{2}^{2}(t)\left|z_{2}(t)-w_{2}(t)\right| \\
\leq & -\left(\theta_{1} b_{1}^{L} \alpha_{1} m_{1}+2 \theta_{1} d_{1}^{L} \alpha_{1}^{2} m_{1}^{2}-\theta_{2} c_{2}^{U} \beta_{1} M_{1}\right)\left|z_{1}(t)-w_{1}(t)\right| \\
& -\left(\theta_{2} b_{2}^{L} \alpha_{2} m_{2}+2 \theta_{2} d_{2}^{L} \alpha_{2}^{2} m_{2}^{2}-\theta_{1} c_{1}^{U} \beta_{2} M_{2}\right)\left|z_{2}(t)-w_{2}(t)\right| \\
= & -s_{1}\left|z_{1}(t)-w_{1}(t)\right|-s_{2}\left|z_{2}(t)-w_{2}(t)\right| \\
\leq & -\sigma \min \left\{1 / \theta_{1}, 1 / \theta_{2}\right\} V(t) \\
= & -\mu V(t),
\end{aligned}
$$

where $\sigma=\min \left\{s_{1}, s_{2}\right\}$ and $\mu=\sigma \min \left\{1 / \theta_{1}, 1 / \theta_{2}\right\}$. It follows from condition (A5) in Theorem 3.1 that we have $\mu>0$, that is, condition (3) in Lemma 2.3 is also satisfied. Therefore, it follows from Lemma 2.3 that system (3.2) has a unique almost periodic solution $\left(z_{1}^{*}(t), z_{2}^{*}(t)\right)$ which is uniformly asymptotically stable in $\mathbb{S}$. That is, system (2.1) has a unique uniformly asymptotically stable positive almost periodic solution $\left(y_{1}^{*}(t), y_{2}^{*}(t)\right)=$ $\left(\exp \left\{z_{1}^{*}(t)\right\}, \exp \left\{z_{2}^{*}(t)\right\}\right)$.

Finally, we will prove that system (1.3) has a unique uniformly asymptotically stable positive almost periodic solution. It follows from Lemma 2.2 that

$$
\left(x_{1}^{*}(t), x_{2}^{*}(t)\right)=\left(\prod_{0<\tau_{k}<t}\left(1+\gamma_{1 k}\right) y_{1}^{*}(t), \prod_{0<\tau_{k}<t}\left(1+\gamma_{2 k}\right) y_{2}^{*}(t)\right)
$$

is a solution of system (1.3). By conditions (A3) and (A4), we can prove that $x_{i}^{*}(t)=$ $\prod_{0<\tau_{k}<t}\left(1+\gamma_{i k}\right) y_{i}^{*}(t)$ is an almost periodic function based on the proofs of Lemma 31 and Theorem 79 in [17]. Thus $\left(x_{1}^{*}(t), x_{2}^{*}(t)\right)$ is a unique uniformly asymptotically stable positive almost periodic solution of system (1.3). The proof of Theorem 3.1 is complete.

\section{An example and numerical simulations}

In this section, to illustrate the feasibility of our analytical results, we give the following example.

Example 4.1 Consider the competitive system with impulsive perturbations

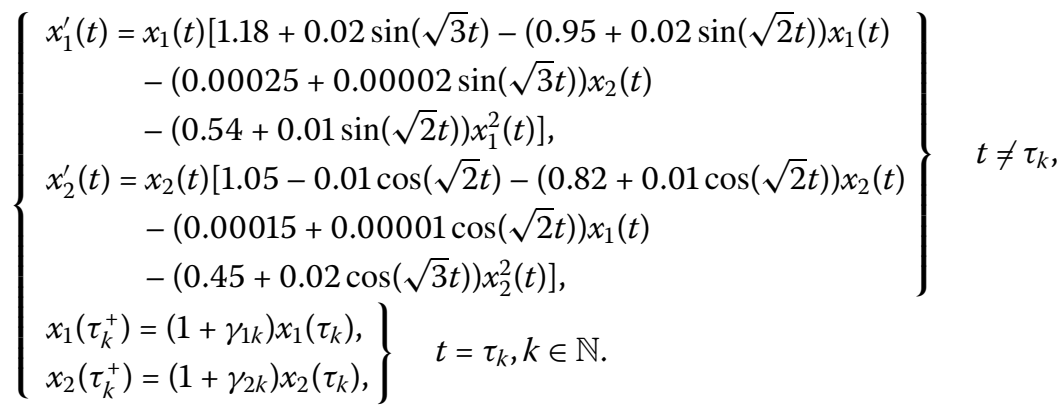


Let $\gamma_{i k}=\exp \left\{(-1)^{k+1} \frac{1}{k^{2}}\right\}-1, \tau_{k}=k, k \in \mathbb{N}$, we obtain that $1<\prod_{0<\tau_{k}<t}\left(1+\gamma_{i k}\right)<e, i=1,2$. So we can choose $\alpha_{1}=\alpha_{2}=1, \beta_{1}=\beta_{2}=e$. A computation shows that

$$
\begin{aligned}
& M_{1} \approx 1.2903, \quad M_{2} \approx 1.3086, \quad m_{1} \approx 0.1471, \quad m_{2} \approx 0.1528, \\
& a_{1}^{L}-c_{1}^{U} \beta_{2} M_{2} \approx 1.1590>0, \quad a_{2}^{L}-c_{2}^{U} \beta_{1} M_{1} \approx 1.0394>0 .
\end{aligned}
$$

Obviously, (A1) and (A2) in Lemma 2.5 are satisfied; moreover, letting $\theta_{1}=\theta_{2}=1$, one has

$$
\begin{aligned}
& s_{1}=\theta_{1} b_{1}^{L} \alpha_{1} m_{1}+2 \theta_{1} d_{1}^{L} \alpha_{1}^{2} m_{1}^{2}-\theta_{2} c_{2}^{U} \beta_{1} M_{1} \approx 0.1592>0, \\
& s_{2}=\theta_{2} b_{2}^{L} \alpha_{2} m_{2}+2 \theta_{2} d_{2}^{L} \alpha_{2}^{2} m_{2}^{2}-\theta_{1} c_{1}^{U} \beta_{2} M_{2} \approx 0.1429>0,
\end{aligned}
$$
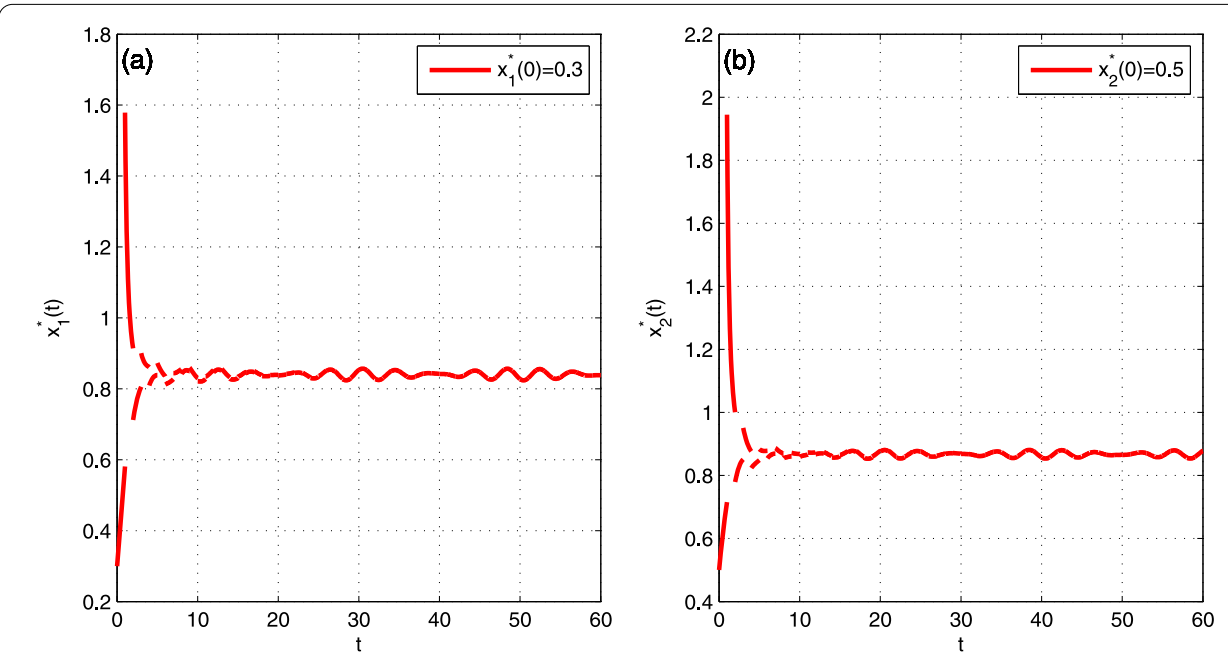

Figure 1 Positive almost periodic solution of system (4.1). (a) Time-series of $x_{1}^{*}(t)$ with initial value $x_{1}^{*}(0)=0.3$ for $t \in[0,60]$. (b) Time-series of $x_{2}^{*}(t)$ with initial value $x_{2}^{*}(0)=0.5$ for $t \in[0,60]$.
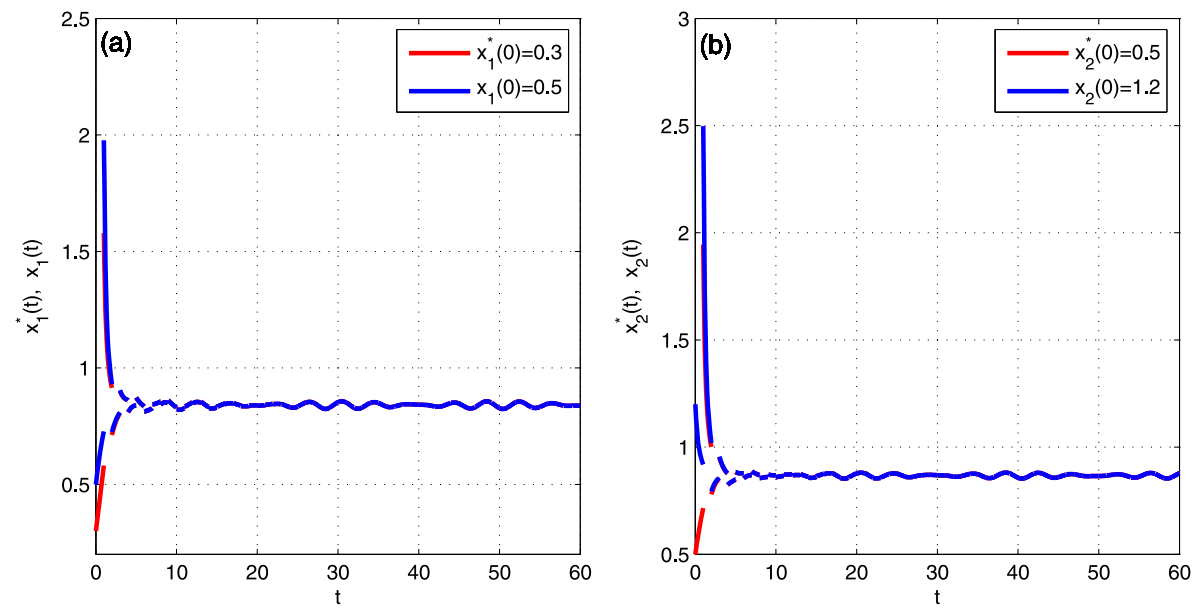

Figure 2 Uniformly asymptotic stability. (a) Time-series of $x_{1}^{*}(t)$ with $x_{1}^{*}(0)=0.3$ and $x_{1}(t)$ with $x_{1}(0)=0.5$ for $t \in[0,60]$, respectively. (b) Time-series of $x_{2}^{*}(t)$ with $x_{2}^{*}(0)=0.5$ and $x_{2}(t)$ with $x_{2}(0)=1.2$ for $t \in[0,60]$, respectively. 
that is, $\sigma=\min \left\{s_{1}, s_{2}\right\} \approx 0.1429>0$, so (A3)-(A5) in Theorem 3.1 are satisfied. Thus, system (4.1) has a unique uniformly asymptotically stable positive almost periodic solution. From Figure 1, we can easily see that system $(4.1)$ with initial value $(0.3,0.5)$ has a positive almost periodic solution denoted by $\left(x_{1}^{*}(t), x_{2}^{*}(t)\right)$. Figure 2 shows that a positive solution with initial value $(0.5,1.2)$, denoted by $\left(x_{1}(t), x_{2}(t)\right)$, tends to the above positive almost periodic solution $\left(x_{1}^{*}(t), x_{2}^{*}(t)\right)$.

\section{Competing interests}

The authors declare that they have no competing interests.

\section{Authors' contributions}

Each of the authors, RT, WL et al. contributed to each part of this work equally and read and approved the final version of the manuscript.

\section{Acknowledgements}

The work is supported by the National Natural Science Foundation of China (No. 11261017) and the Project of Key Laboratory of Biological Resources Protection and Utilization of Hubei Province (Forestry). We would like to thank anonymous reviewers for their helpful comments which improved the presentation of this work.

Received: 6 October 2013 Accepted: 3 December 2013 Published: 02 Jan 2014

\section{References}

1. Gopalsamy, K: Stability and Oscillations in Delay Differential Equation of Population Dynamics. Kluwer Academic, Dordrecht (1992)

2. Samanta, GP: A two-species competitive system under the influence of toxic substances. Appl. Math. Comput. 216, 291-299 (2010)

3. Jin, Z, Han, MA, Li, GH: The persistence in a Lotka-Volterra competition systems with impulsive. Chaos Solitons Fractals 24, 1105-1117 (2005)

4. Liu, ZJ, Wu, JH, Tan, RH: Permanence and extinction of an impulsive delay competitive Lotka-Volterra model with periodic coefficients. IMA J. Appl. Math. 74, 559-573 (2009)

5. Li, JX, Yan, JR: Partial permanence and extinction in an N-species nonautonomous Lotka-Volterra competitive system. Comput. Math. Appl. 55, 76-88 (2008)

6. Hou, J, Teng, ZD, Gao, SJ: Permanence and global stability for nonautonomous N-species Lotka-Volterra competitive system with impulses. Nonlinear Anal., Real World Appl. 11, 1882-1896 (2010)

7. Montes de Oca, F, Vivas, M: Extinction in a two dimensional Lotka-Volterra system with infinite delay. Nonlinear Anal., Real World Appl. 7, 1042-1047 (2006)

8. Liu, ZJ, Fan, M, Chen, LS: Globally asymptotic stability in two periodic delayed competitive systems. Appl. Math. Comput. 197, 271-287 (2008)

9. Tang, XH, Cao, DM, Zou, XF: Global attractivity of positive periodic solution to periodic Lotka-Volterra competition systems with pure delay. J. Differ. Equ. 228, 580-610 (2006)

10. Liu, B, Chen, LS: The periodic competing Lotka-Volterra model with impulsive effect. Math. Med. Biol. 21, 129-145 (2004)

11. Fan, $M$, Wang, K, Jiang, DQ: Existence and global attractivity of positive periodic solutions of periodic $n$-species Lotka-Volterra competition systems with several deviating arguments. Math. Biosci. 160, 47-61 (1999)

12. Gopalsamy, K: Global asymptotic stability in an almost periodic Lotka-Volterra system. J. Aust. Math. Soc. B 27, 346-360 (1986)

13. Ahmad, S: On almost periodic solutions of the competing species problem. Proc. Am. Math. Soc. 102, $855-861$ (1988)

14. He, MX, Chen, FD, Li, Z: Almost periodic solution of an impulsive differential equation model of plankton allelopathy. Nonlinear Anal., Real World Appl. 11, 2296-2301 (2010)

15. Ahmad, S, Stamov, GT: Almost periodic solutions of dimensional impulsive competitive systems. Nonlinear Anal., Real World Appl. 10, 1846-1853 (2009)

16. Zhang, TW, Li, YK, Ye, Y: On the existence and stability of a unique almost periodic solution of Schoener's competition model with pure-delays and impulsive effects. Commun. Nonlinear Sci. Numer. Simul. 17, 1408-1422 (2012)

17. Samoilenko, AM, Perestyuk, NA: Impulsive Differential Equations. World Scientific, Singapore (1995)

18. Bainov, DD, Simenov, PS: Impulsive Differential Equations: Periodic Solutions and Applications. Longman, Harlow (1993)

19. Lakshmikantham, V, Bainov, DD, Simeonov, PS: Theory of Impulsive Differential Equations. World Scientific, Singapore (1989)

20. He, CY: Almost Periodic Differential Equations. Higher Education Press, Beijing (1992) (Chinese version)

21. Chen, FD, Li, Z, Huang, YJ: Note on the permanence of a competitive system with infinite delay and feedback controls. Nonlinear Anal., Real World Appl. 8, 680-687 (2007)

10.1186/1687-1847-2014-2

Cite this article as: Tan et al.: Uniformly asymptotic stability of almost periodic solutions for a competitive system with impulsive perturbations. Advances in Difference Equations 2014, 2014:2 